\title{
Effects of solar wind speed on the secondary energetic neutral source of the Interstellar Boundary Explorer ribbon
}

\author{
E. J. Zirnstein ${ }^{1}$, H. O. Funsten ${ }^{2}$, J. Heerikhuisen ${ }^{3}$, and D. J. McComas ${ }^{1,4}$ \\ ${ }^{1}$ Southwest Research Institute, 6220 Culebra Road, San Antonio, TX 78238, USA \\ e-mail: ezirnstein@swri.edu \\ 2 Los Alamos National Laboratory, PO Box 1663, Los Alamos, NM 87545, USA \\ 3 Department of Space Science, University of Alabama in Huntsville, 301 Sparkman Drive, Huntsville, AL 35899, USA \\ ${ }^{4}$ Department of Physics and Astronomy, University of Texas at San Antonio, 1 UTSA Circle, San Antonio, TX 78249, USA
}

Received 23 September 2015 / Accepted 5 November 2015

\begin{abstract}
The Interstellar Boundary EXplorer (IBEX) ribbon is an intense energetic neutral atom (ENA) emission feature encircling the sky, spanning energies $\leq 0.5-6 \mathrm{keV}$. The ribbon may be produced by the "secondary ENA" mechanism, where ENAs emitted from a source plasma population inside the heliosphere propagate outside the heliopause, undergo two charge-exchange events, and become secondary ENAs that may be directed back toward Earth and detected by IBEX. In this scenario, the source plasma population is governed by the interaction of the solar wind (SW) with the interstellar medium and is thus sensitive to the global SW properties. Moreover, this scenario predicts that the distance to the source of secondary ENAs depends on the ENA energy and SW speed, which in turn may affect the shape of the ribbon. In this paper, we use a computational model of the heliosphere with simplified SW boundary conditions to analyze the influence of ENA energy and SW speed, independent of time and latitude, on the global spatial and geometric properties of the ribbon. We find a strong dependence of the simulated ribbon energy spectrum and spatial symmetry on SW speed and ENA energy, and only a slight dependence on ribbon geometry. Our results suggest a significant number of primary ENAs from the inner heliosheath may contribute to the pickup ion source population outside the heliopause, depending on the ENA energy and SW speed. The lack of variation in the simulated ribbon center as a function of ENA energy and SW speed, in contrast to the observations, implies that the asymmetry of the SW plays an important role in determining the position of the ribbon. Comparisons to the IBEX data also signify the ribbon's dependence on the properties of the local interstellar medium, particularly the interstellar magnetic field.
\end{abstract}

Key words. Sun: heliosphere - solar wind - ISM: atoms - ISM: magnetic fields

\section{Introduction}

The Interstellar Boundary EXplorer (IBEX) discovered the "ribbon" of enhanced energetic neutral atom (ENA) emission in the sky (McComas et al. 2009). It was shown to have a strong intensity compared to the globally distributed flux, with an average width of $\sim 20^{\circ}$ (Fuselier et al. 2009). The ribbon is nearly circular in shape (Funsten et al. 2009b, 2013) with an average radius $\sim 74.5^{\circ}$, and it is approximately centered on ecliptic (J2000) coordinates $\left(219.2^{\circ}, 39.9^{\circ}\right)$ (Funsten et al. 2013). The distribution of ENA flux around the circular ribbon appears symmetric with unimodal symmetry at $\sim 1 \mathrm{keV}$ and bimodal symmetry at higher energies (Funsten et al. 2015). The IBEX ribbon is presumably ordered by the interstellar magnetic field; a comparison of the ribbon structure to global magneto-hydrodynamic (MHD) models of the heliosphere showed that the ribbon flux approximately aligns with directions satisfying $\boldsymbol{B} \cdot \boldsymbol{r}=0$ (Schwadron et al. 2009a), where $\boldsymbol{B}$ is the local interstellar medium (LISM) magnetic field that is draped around the heliopause, and $\boldsymbol{r}$ is the radial line-of-sight view from the inner heliosphere and thus of IBEX.

Even from the initial discovery of the IBEX ribbon, many possible mechanisms have been suggested for its source, spanning from the termination shock, through the compressed and heated inner heliosheath (IHS) to the heliopause, and out into the compressed and heated outer heliosheath (OHS) in the interstellar medium beyond the heliopause (McComas et al. 2009). Since then, many of these have been developed into quantitative models, while other, completely new ideas have also arisen. Various models include sources near the termination shock (Kucharek et al. 2013), in the IHS (Siewert et al. 2013; Fichtner et al. 2014; see also the quantitative calculation of the hydrogen (H)-wave scenario in Sylla \& Fichtner 2015), in the OHS (Heerikhuisen et al. 2010b; Chalov et al. 2010; Schwadron \& McComas 2013), or in the distant LISM (Grzedzielski et al. 2010). For a review of hypothesized ribbon mechanisms, see McComas et al. (2014) and references therein.

The secondary ENA mechanism is one of the original proposed mechanisms for the origin of the ribbon (McComas et al. 2009). As the cold, slow neutral interstellar gas traverses the heliosphere, interstellar neutral atoms, mainly $\mathrm{H}$, charge exchange with fast solar wind (SW) ions. An ionized, cold neutral atom is "picked up" by the motional electric field of the bulk SW plasma (i.e., pickup ion, PUI), and the neutralized SW ion (called "primary" ENA to distinguish this first charge-exchange interaction) follows a ballistic trajectory that is not influenced by ambient electric or magnetic fields. The primary ENA can easily traverse the heliosphere due to its large mean free path and can cross the heliopause into the dense OHS. Over time, the ENA that was originally a SW ion can undergo two sequential charge-exchange events (ionization followed by a second neutralization) in the 
OHS, creating a "secondary" ENA that may be directed back toward the inner heliosphere. Of critical importance is the interaction dynamics of the ionized ENA in the OHS, specifically its pitch angle relative to the interstellar magnetic field: near $90^{\circ}$ pitch angle, ionized ENAs will gyrate at nearly a fixed location in the sky, and subsequent secondary neutralization yields emission concentrated along radial directions perpendicular to the interstellar magnetic field that drapes around the heliopause, i.e., $\boldsymbol{B} \cdot \boldsymbol{r}=0$, that can be observed in the inner heliosphere (e.g., by IBEX).

Since the first IBEX observations, simulations and models based on the secondary ENA mechanism were able to qualitatively and quantitatively reproduce many aspects of the IBEX ribbon (Heerikhuisen et al. 2010b, 2014; Chalov et al. 2010; Heerikhuisen \& Pogorelov 2011; Schwadron \& McComas 2013; Möbius et al. 2013; Zirnstein et al. 2013, 2015b,a; Isenberg 2014; Giacalone \& Jokipii 2015). The details of the parent PUI dynamics in the OHS, however, are still under debate (also see Florinski et al. 2010; Gamayunov et al. 2010; Liu et al. 2012; Summerlin et al. 2014; Zank et al. 2014).

Here we investigate the effects of different SW boundary conditions, uniform in latitude, on the IBEX ribbon intensity and structure based on the secondary ENA mechanism. According to this mechanism, the neutralized SW provides primary ENAs that generate the parent PUI population in the OHS. Assuming these PUIs do not change significantly in energy (i.e., no diffusion in velocity space), and PUI scattering is largely independent of the energies we consider $(\sim 1 \mathrm{keV})$, the properties of the SW will largely determine the intensity and structure of the ribbon flux as a function of energy and latitude as shown in the IBEX observations by McComas et al. (2012b). The properties of the LISM will also affect the location of the ribbon (e.g., Heerikhuisen \& Pogorelov 2011; Grygorczuk et al. 2011; Strumik et al. 2011; Ratkiewicz et al. 2012; Ben-Jaffel \& Ratkiewicz 2012; Ben-Jaffel et al. 2013), and its intensity and structure (e.g., Frisch et al. 2010; Heerikhuisen et al. 2014). However, in this paper we focus solely on the influence of the $\mathrm{SW}$ on the ribbon.

Several studies have modeled the effects of the latitudinal structure of the SW on the secondary ENA emission that forms the ribbon. Based on the "partial shell" approximation for the PUI distribution in the OHS (see Sect. 2.2), Heerikhuisen et al. (2010a) applied a simplified model of the dynamic SW to their simulations of the IBEX ribbon, based on SW data derived from Ulysses observations (Ebert et al. 2009). ENAs generated by charge exchange in the supersonic SW in their kinetic-neutral code were given speeds scaled by observed SW speeds picked randomly over a solar cycle. Heerikhuisen et al. (2010a) produced a ribbon spectrum with a "bump" in intensity that was spread over the slow and fast SW energies, a more realistic scenario compared to previous simulations assuming uniform solar maximum conditions. Heerikhuisen et al. (2012) analyzed the ribbon spectra in an idealized simulation of the $11 \mathrm{yr}$ solar cycle similar to Pogorelov et al. (2009), although assuming instantaneous propagation of ENAs from the source to $1 \mathrm{au}$. They found that the ribbon "knot", a small structure of enhanced intensity at high latitudes seen in the IBEX data (McComas et al. 2009, 2010), also formed in their simulation and that it showed considerable variation over the solar cycle with a smaller spectral index produced from the fast SW at solar minimum, and a larger spectral index at solar maximum. Schwadron \& McComas (2013), Heerikhuisen et al. (2014), Zirnstein et al. (2013), and Zirnstein et al. (2015a) included SW conditions derived from in situ and interplanetary scintillation measurements of the SW (Sokół et al. 2013), reproducing the latitudinal profile of the IBEX ribbon as a function of energy. Zirnstein et al. (2015b) analyzed the effects of a dynamic solar cycle on the ribbon flux with self-consistent propagation of ENAs from the source to $1 \mathrm{au}$, finding a quasi-periodic variation of the ribbon flux with a period similar to, but out of phase with, the $11 \mathrm{yr}$ solar activity cycle.

While Zirnstein et al. (2015b) currently provides the most sophisticated model of the time-dependent ribbon, this model introduces multiple variants into the analysis of the ribbon that are difficult to interpret simultaneously, such as the time dependence of the SW, line-of-sight integration of evolving plasma/neutral distributions, latitudinal asymmetry of the SW, and the inherent time delays of SW/ENA propagation and charge exchange. Here we provide a simpler, systematic study of the SW driver, and analyze and compare the effects of SW with similar dynamic pressures, but different speeds and densities, on the intensity and structure of the ribbon. In particular, we study the dependence of the structure and spectral geometry of the ribbon on the SW speed and ENA energy. The speed of the SW, and thus energy of primary and secondary ENAs, may affect the average distance from the heliopause at which the majority of ribbon ENAs (observable at $1 \mathrm{au}$ ) originate. By removing the above-mentioned variants, we can more closely focus on the relationship between SW speed, ENA energy, and the ribbon. The results of this paper will help us understand IBEX observations of the ribbon as well as shed insight into models of the secondary ENA process. The paper is organized as follows. In Sect. 2 we describe the SW boundary conditions, the ribbon flux simulation, and analysis procedures. In Sect. 3 we present the results of our simulations, and compare these to current IBEX observations. Finally, in Sect. 4 we discuss the implications of the results.

\section{Simulation and analysis procedure}

Our simulation process involves two parts. In the first part (Sect. 2.1), we solve the coupled MHD and neutral dynamics equations to obtain a steady-state solution of the heliosphere. In the second part (Sect. 2.2), we perform a sophisticated postprocessing technique that uses the simulated plasma distribution and the 6D neutral phase space distribution to integrate the sources of ENAs at a given energy along all IBEX look directions.

\subsection{Simulation of the heliosphere}

We simulate the SW-LISM interaction using a 3D MHDplasma/kinetic-neutral code (Heerikhuisen et al. 2006a,b, 2009; Pogorelov et al. 2008). We assume an ideal MHD, single-fluid description of the plasma, which is represented by a generalized Lorentzian, or "kappa" ( $\kappa)$, distribution (e.g., Vasyliunas 1968; Christon et al. 1989; Treumann 1999; Livadiotis \& McComas 2009, 2013) in the IHS, and Maxwellian elsewhere. This allows us to approximate the SW as the core of the distribution, and PUIs and suprathermal ions in the high energy tail of the distribution in the hot IHS. We assume $\kappa=1.63$ throughout the IHS, which has been used in multiple studies when comparing simulations of H ENA flux from the IHS with IBEX observations (e.g., Zank et al. 2010; Desai et al. 2012, 2014; Zirnstein et al. 2014). The neutral $\mathrm{H}$ atoms are modeled kinetically by solving the Boltzmann transport equation, and are coupled to the plasma by charge exchange through mass, momentum, and energy source terms (Heerikhuisen et al. 2006b, 2009). The MHDplasma and kinetic-neutral modules are iteratively repeated until 
Table 1. SW boundary conditions at 1 au.

\begin{tabular}{lccc}
\hline \hline Parameters & Slow & Intermediate & Fast \\
\hline Plasma density, $n_{\mathrm{p}}\left(\mathrm{cm}^{-3}\right)$ & 5.74 & 2.77 & 1.8 \\
Speed, $v\left(\mathrm{~km} \mathrm{~s}^{-1}\right)$ & 400 & 600 & 775 \\
Temperature, $T(\mathrm{~K})$ & 74000 & 152000 & 230000 \\
Field magnitude, $B_{\mathrm{r}}(\mu \mathrm{G})$ & 22.3 & 22.3 & 22.3 \\
Ram pressure, $m_{\mathrm{p}} n_{\mathrm{p}} v^{2}(\mathrm{nPa})$ & 1.54 & 1.67 & 1.81 \\
\hline
\end{tabular}

a steady-state solution of the heliosphere is reached. The results of plasma and neutral $\mathrm{H}$ atom properties are collected in spherical grids, which are then used to compute H ENA fluxes at 1 au (see Sect. 2.2).

We simulate the heliosphere separately for three sets of SW conditions: (1) slow SW; (2) intermediate speed SW; and (3) fast SW, all of which are assumed to be independent of latitude and time. The SW conditions at the simulation inner boundary $(10 \mathrm{au})$ are computed by propagating the SW conditions at 1 au to 10 au assuming adiabatic expansion. The conditions at 1 au are listed in Table 1 for each case, which are based on in situ observations. The value of $B_{\mathrm{r}}$ in Table 1 is lower than our previous simulations $\left(B_{\mathrm{r}}=37.5 \mu \mathrm{G}\right.$, e.g., Zirnstein et al. 2013; Heerikhuisen et al. 2014). Here we use a value derived from SW data averaged between 2009 and 2013 (McComas et al. 2013a), which reflects more recent SW conditions. We also note that we use the same value of $B_{\mathrm{r}}$ for the slow, intermediate, and fast SW speed cases. $B_{\mathrm{r}}$ experiences solar cycle variations (e.g., Zhou \& Smith 2009); however, magnetic flux $\left(r^{2} B_{\mathrm{r}}\right)$ is approximately independent of latitude (Smith et al. 2001). Since our model is not time dependent, and we are mainly concerned with the relationship between the ribbon and SW speed, for simplicity we use the same value of $B_{\mathrm{r}}$ for each case. The intermediate SW conditions are chosen in order to produce a ram pressure equal to the average of the fast and slow SW pressures. The differences between ram pressures in all cases are between $\sim 8 \%$ and $16 \%$, consistent with the observation of ram pressure nearly independent of latitude (McComas et al. 2008, 2013a).

The LISM outer boundary conditions at 1000 au are the same as those used by Heerikhuisen et al. (2014) in their $B=3 \mu \mathrm{G}$ LISM magnetic field simulation case, where plasma density $n_{\mathrm{p}}=0.082 \mathrm{~cm}^{-3}$, neutral density $n_{\mathrm{H}}=0.172 \mathrm{~cm}^{-3}$, inflow speed $v=23.2 \mathrm{~km} \mathrm{~s}^{-1}$, temperature $T=6300 \mathrm{~K}$, and inflow direction is ecliptic $\left(259^{\circ}, 5^{\circ}\right)$ (Bzowski et al. 2012; Möbius et al. 2012; McComas et al. 2012a). With a strength of $3 \mu \mathrm{G}$, the simulation's interstellar magnetic field direction is assumed to be aligned with the IBEX ribbon center (Funsten et al. 2013), with a pristine direction toward ecliptic $\left(219.2^{\circ}, 39.9^{\circ}\right)$.

Recent analyses of IBEX observations of the LISM over the last six years (see McComas et al. 2015, and references therein) show that the interstellar flow speed, direction, and temperature are slightly different compared to the values we use in our simulations. This evidence was not available before a significant portion of the simulation and analysis for this paper was complete. Our experience with simulations of this type suggests that the ribbon flux and geometry will be only slightly affected, and that our conclusions regarding the comparison of the three cases we consider would not change.

\subsection{Simulating the IBEX ribbon}

Once we have solved for the SW-LISM interaction, we simulate the IBEX ribbon flux using the partial shell approximation of PUIs in the OHS (Heerikhuisen et al. 2010b; Zirnstein et al. 2012). This treats the parent PUIs as a partial shell distribution, where the angular width of the shell depends on the angle between the outgoing, primary ENA and the LISM magnetic field direction. For example, ENAs that propagate outward nearly perpendicular to the LISM magnetic field will, upon charge exchange, produce a ring-like PUI distribution strongly concentrated near $90^{\circ}$ pitch angle. This method allows us to produce a ribbon intensity that peaks near directions toward $\boldsymbol{B} \cdot \boldsymbol{r}=0$, and diminishes farther away. The dynamics of PUIs in the OHS are likely more complicated, such that parallel-propagating PUIs may not scatter as easily as PUIs with large pitch angles (e.g., Schwadron \& McComas 2013; Isenberg 2014), PUIs produced near $\boldsymbol{B} \cdot \boldsymbol{r}=0$ may scatter onto nearly full shell distributions (Florinski et al. 2010; Schwadron \& McComas 2013; Isenberg 2014), PUIs may experience little to no net scattering (Chalov et al. 2010; Möbius et al. 2013), or become trapped in preexisting LISM magnetic field turbulence (Giacalone \& Jokipii 2015), depending on the behavior of the PUIs and the level of turbulence in the OHS. For example, recent observations by Voyager 1 suggest that the OHS has unexpectedly low levels of turbulence (Burlaga et al. 2014, 2015; Burlaga \& Ness 2014). Since our goal is to study the effect of SW properties on the geometry of the ribbon, which is largely determined by the directions toward $\boldsymbol{B} \cdot \boldsymbol{r}=0$, our approach is sufficient for this paper.

To compute the ENA emission flux from the ribbon, we integrate the differential, secondary ENA flux equation (Zirnstein et al. 2012, 2013), given by

$$
\begin{aligned}
\Delta J(\boldsymbol{r}, v)= & \frac{1}{4 \pi m_{\mathrm{H}}} n_{\mathrm{p}}(\boldsymbol{r}) v^{2} P(\boldsymbol{r}, v) \\
& \times\left[\int_{\Omega_{v}} \frac{f_{\mathrm{H}}\left(\boldsymbol{r}, \boldsymbol{v}_{\mathrm{H}}\right)}{\hat{v}_{\mathrm{H}} \cdot \hat{B}} v_{\mathrm{rel}}\left(\left|\boldsymbol{v}_{\mathrm{H}}-\boldsymbol{u}_{\mathrm{p}}\right|\right) \sigma_{\mathrm{ex}}\left(v_{\mathrm{rel}}\right) \mathrm{d} \Omega_{v}\right] \Delta t,
\end{aligned}
$$

where $m_{\mathrm{H}}$ is the mass of $\mathrm{H}, n_{\mathrm{p}}$ is the proton density, $v$ is the ENA speed, $P$ is survival ENA probability to 100 au (only including losses due to charge exchange outside $100 \mathrm{au}), f_{\mathrm{H}}$ is the $6 \mathrm{D} \mathrm{H}$ distribution integrated over velocity $\boldsymbol{v}_{\mathrm{H}}$ (where $v_{\mathrm{H}}=$ $v$ ) in velocity space $\Omega_{v}$ (Heerikhuisen \& Pogorelov 2010), $\boldsymbol{B}$ is the interstellar magnetic field vector, $v_{\text {rel }}$ is the relative velocity between parent ENA and bulk plasma computed using the equations from Ripken \& Fahr (1983; although corrected for a typographical error), $\sigma_{\mathrm{ex}}$ is the energy-dependent, charge-exchange cross section (Lindsay \& Stebbings 2005), and $\Delta t$ is the integration time step. Equation (1) is integrated by interpolating local plasma and neutral results from the MHD/kinetic simulation of the heliosphere along $\mathrm{H}$ ENA trajectories that coincide with IBEX lines-of-sight. The contribution of the partial shell distribution of PUIs to the ENA flux visible at 1 au is determined by the denominator term in the integral in Eq. (1), where the angle between the primary ENA velocity vector and the interstellar magnetic field vector determines the intensity of the resulting secondary ENA flux (i.e., smaller angle produces higher intensity since ENAs are assumed to occupy a smaller fraction of a shell in velocity space). However, if the line-of-sight view from 1 au does not intersect the partial shell distribution of PUIs (Heerikhuisen et al. 2010b; Zirnstein et al. 2012), then ENAs produced from those PUIs cannot be observed by IBEX and are not counted in the flux seen at $1 \mathrm{au}$.

As was done in our previous work (Zirnstein et al. 2013, 2015b; Heerikhuisen et al. 2014), we simulate the IBEX ribbon flux at all IBEX-Hi energies, integrating over a discrete set of energies in each IBEX-Hi energy passband, and weight the contribution of flux at each discrete energy using the IBEX-Hi 
energy response functions (Funsten et al. 2009a; Schwadron et al. 2009b). Each pixel in the simulated all-sky map is filtered using a simplified model of the IBEX-Hi hexagonal collimator response function (Funsten et al. 2009a; Zirnstein et al. 2013), where we assume a full width at half maximum (FWHM) of $7^{\circ}$ (IBEX-Hi's nominal FWHM is $\sim 6.5^{\circ}$ ). This tends to smooth sharp features in the sky. The results of these simulations are presented in Sect. 3.

\section{Results and discussion}

\subsection{All-sky maps of ribbon flux}

In this section we present our results of simulating the IBEX ribbon in each IBEX-Hi energy passband for the uniform slow, intermediate, and fast SW boundary conditions listed in Table 1. We briefly describe the general features of the ribbon, and more carefully analyze the structure and geometry of the ribbon in Sect. 3.2 (for a more sophisticated model of the time-dependent, secondary ENA ribbon and its comparison to IBEX observations, see Zirnstein et al. 2015b). All-sky maps of ribbon ENA flux are shown in Fig. 1. The color bar scales are the same as those used by Schwadron et al. (2014) in their separated ribbon results.

In general, at low ENA energies, the brightest ENA emission is seen for the slow SW case; in contrast, at high ENA energies the brightest emission results from the fast SW case. Comparing to Fig. 2 of Schwadron et al. (2014), for the case of slow SW the simulated ribbon flux overestimates the observed flux below $1.11 \mathrm{keV}$, but underestimates above $1.11 \mathrm{keV}$. For the case of intermediate speed SW, we see the simulated ribbon flux is overestimated at $1.74 \mathrm{keV}$ and $2.73 \mathrm{keV}$, produces results similar to observation at $1.11 \mathrm{keV}$, and is underestimated at the lowest and highest energies. For the case of fast SW, we see an enhancement in flux above $1.74 \mathrm{keV}$. Because the SW speeds are uniform in latitude at the inner boundary, there is generally no bimodal structure seen in the simulated ENA intensity, even at high energies (Funsten et al. 2015). This can be seen by comparing to the separated IBEX ribbon flux in Fig. 2 of Schwadron et al. (2014), which show a clear dimming of ribbon flux at low latitudes above $\sim 2 \mathrm{keV}$, relative to the high-latitude flux. Initial comparisons between the results and IBEX data suggest (1) SW with speeds between slow $\left(\sim 400 \mathrm{~km} \mathrm{~s}^{-1}\right)$ and intermediate $\left(\sim 600 \mathrm{~km} \mathrm{~s}^{-1}\right)$ must exist at low to mid latitudes to reproduce the ribbon at most energies; (2) the $2.73 \mathrm{keV}$ ribbon shows that most of the SW below $+30^{\circ}$ latitude is between slow and intermediate speeds, with intermediate to fast $\left(\sim 775 \mathrm{~km} \mathrm{~s}^{-1}\right) \mathrm{SW}$ near the "knot" $\left(+60^{\circ}\right.$ latitude); and (3) no single SW speed can reproduce the IBEX ribbon at all energies. This highlights the importance of the latitudinal distribution of the SW over the epoch of IBEX observations (beginning of 2009; McComas et al. 2012b).

In the next few years, temporal dynamics driven by solar maximum, which includes disappearance of the large polar coronal holes and thus reduction of fast SW $\left(>700 \mathrm{~km} \mathrm{~s}^{-1}\right)$ appearing at high latitudes, present a key IBEX observational test for the secondary ENA ribbon hypothesis. While we expect many of the suggested ribbon scenarios to be driven by the dynamics of the SW, it may be possible to discriminate between different ribbon scenarios based on their source region and governing dynamics, such as the evolution of the parent proton distribution, the processing that occurs between the creation of the parent proton and ENA detected by IBEX, and the time it takes for changes in the SW to be reflected in ENAs. However, smaller and more transitory coronal holes appear at all latitudes, creating a complicated mixture of slow to fast SW (McComas et al. 2008, 2013a). This increase in complexity makes a detailed comparison between our results and IBEX observations challenging. On the other hand, McComas et al. (2012b) predicted that this mixture of fast and slow winds at all latitudes around solar maximum would produce a ribbon where the energies of the observed ENAs would be far less latitude dependent than they were in the first five years of IBEX observations, resulting in observations more closely related to our simulation results. Therefore, given the proper time delays for SW and ENA propagation, our simulations predict the high energy ENA ribbon flux will drop significantly, as can be seen at $2.73 \mathrm{keV}$, and especially at $4.29 \mathrm{keV}$, in our Fig. 1 (see also McComas et al. 2012b, 2014). At low ENA energies $(0.71$ and $1.11 \mathrm{keV})$ with predominantly slow SW, we expect to see an enhancement in ENA intensity at high latitudes, especially at $0.71 \mathrm{keV}$. The ribbon at $1.74 \mathrm{keV}$ is an interesting case, as it reaches a maximum between the cases of slow and fast SW, and its evolution over time may be more complicated. The ribbon symmetry at $1.74 \mathrm{keV}$ appears to be a transitional energy between unimodal and bimodal flux distributions (Funsten et al. 2015, Fig. 7) and is additional evidence of a mixture of structures and/or processes. However, for a more detailed study of the influence of the dynamic SW on the secondary ENA ribbon, see Zirnstein et al. (2015b).

\subsection{Circularity results}

In this section we look at how the SW speed and ENA energy are related to the structure and geometry of the secondary ENA ribbon. In Fig. 2 we show an example of the simulated ribbon flux rotated to a common ribbon-centered frame. In this frame we derive key geometrical properties, such as the ribbon center, radius, eccentricity, and rotation angle, similar to the analysis of Funsten et al. (2013).

\subsubsection{Spatial asymmetry in simulated ribbon intensity}

Before discussing the results of the circularity analysis, we note a few interesting features that are seen in Fig. 1. First, at low ENA energies $(\sim 0.71-1.11 \mathrm{keV})$, ribbon flux originating from the slow SW peaks near the nose of the heliosphere, and drops off almost symmetrically away from the nose. This is likely due to the fact that the majority of interstellar neutral atoms charge exchange upstream of the Sun, producing more primary ENAs, with speeds near the bulk SW speed, that propagate outward toward the front of the heliosphere, and may come back inward as secondary ENAs (for a 3D view of the secondary ENA source of the ribbon, see Zirnstein et al. 2015a). This behavior is seen near $\sim 1 \mathrm{keV}$ during slow SW, near $\sim 2 \mathrm{keV}$ during intermediate speed SW, and near $\sim 3-4 \mathrm{keV}$ during fast SW. This can be seen even more clearly in Fig. 3, where we have plotted the simulated ribbon flux (left panels, solid curves, left $y$-axis) as a function of azimuthal angle located along the elliptical fits to the simulated ribbon flux at each energy, as demonstrated in Fig. 2 (i.e., locations of maximum ribbon flux). Angle $0^{\circ}$ (on the $+x$-axis) intersects the LISM field-nose plane ( $B-V$ plane), and $-180^{\circ}$ to $+180^{\circ}$ runs counterclockwise. We have also plotted the heliolatitude coordinates of the ribbon location (dashed curves, right $y$-axis) defined by the elliptical fits demonstrated in Fig. 2.

Conversely, for faster SW the low energy ribbon flux drops significantly near the front of the heliosphere, but flux closer to the heliotail remains relatively unchanged. This behavior introduces an important property of the secondary ENA process that 
E. J. Zirnstein et al.: Effects of solar wind speed on energetic neutrals from the IBEX ribbon
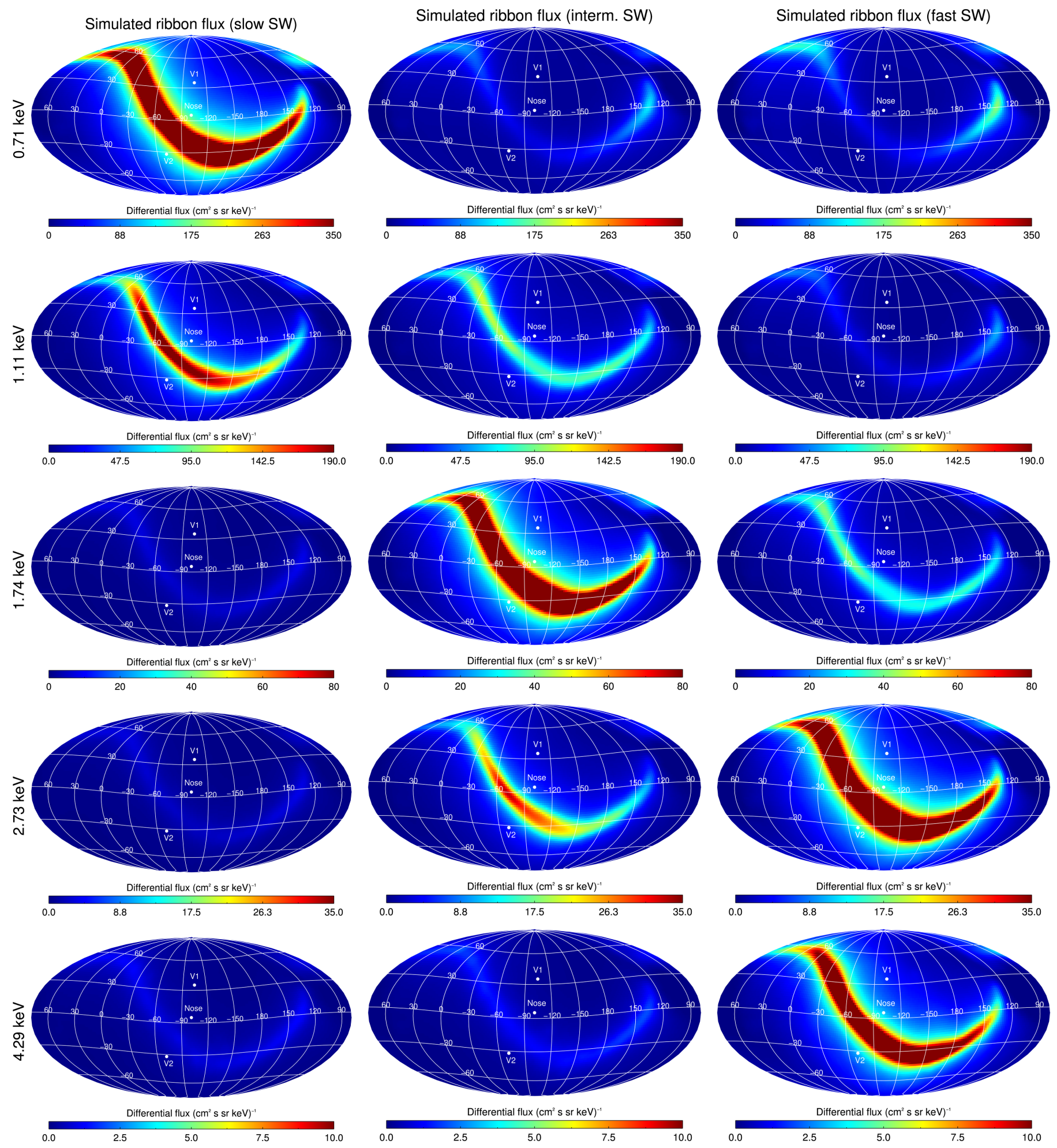

Fig. 1. All-sky maps of simulated ribbon flux, centered on the LISM inflow direction (i.e., Nose). We show results for the uniform slow (left column), intermediate (middle column), and fast (right column) SW, for IBEX-Hi energy passbands 2-6 (top-down). The directions toward the nose, Voyager 1 (V1), and Voyager 2 (V2) are shown. Note the color bar scales are the same for each SW case, but different for each energy. The color scales are the same as those used in Fig. 2 of Schwadron et al. (2014).

has not yet been quantified: the relative contribution of outwardpropagating, primary ENAs from the supersonic SW versus the IHS to the secondary ENA ribbon source. The IHS plasma contributes some primary ENAs to the OHS PUI population, mostly concentrated outside the heliotail. At low ENA energies, the component of the ribbon originating from outward-propagating
ENAs born in the IHS is drowned out by the source from the slow, neutralized supersonic SW. However, during faster SW the component of ENAs from the supersonic SW at low energies drops significantly, revealing the component of the ribbon from ENAs born in the IHS. This behavior can be seen in the right panels of Fig. 3, where we have separated the ribbon 


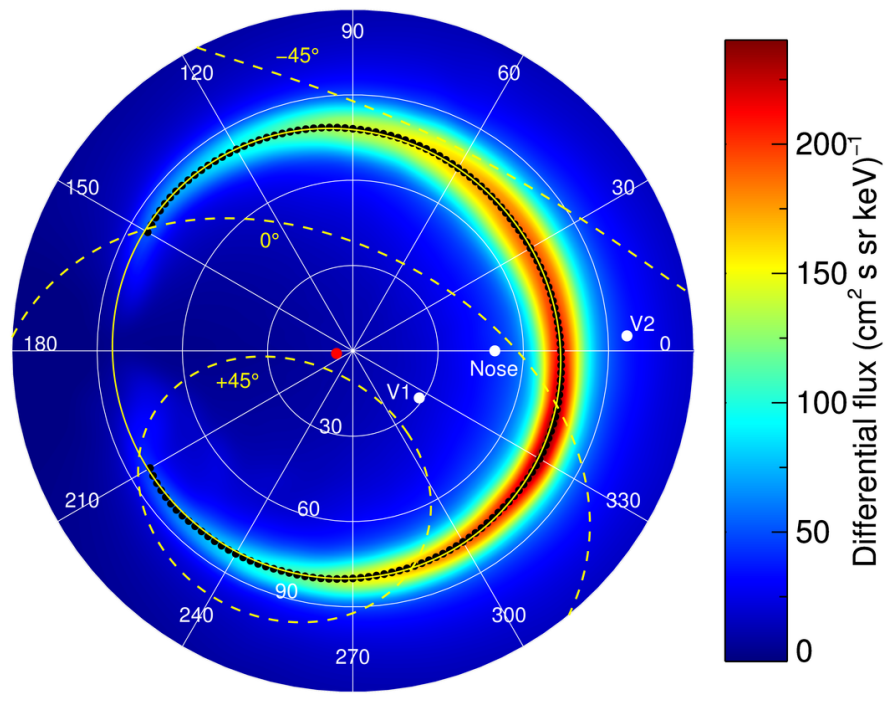

Fig. 2. Simulated ribbon flux plotted in ribbon-centered coordinate system similar to Funsten et al. (2013), for energy passband 3, slow SW case. The map is centered on $\left(219.2^{\circ}, 39.9^{\circ}\right)$ in ecliptic coordinates and the heliospheric nose lies on the $+x$ axis. For this study, we assume that the interstellar magnetic field is aligned with the IBEX ribbon center; the $x$-axis therefore defines the so-called $B-V$ plane that contains both the interstellar magnetic field vector and the direction of motion of the Sun through the ISM. We show the ribbon flux maxima along the ribbon (black dots), the best-fit ellipse to the maxima (yellow solid curve), and the ellipse center (red dot). We also show the heliolatitude lines at $+45^{\circ}, 0^{\circ}$, and $-45^{\circ}$ as yellow dashed curves.

flux into different components depending on their origination. Other effects, such as changes in the mean free path of interstellar neutrals in the inner heliosphere as the SW changes, may also contribute to the evolution of the ribbon intensity, but do not appear to be as important as understanding the source of the primary ENAs to the OHS PUI population. We note that our assumption of a constant $\kappa=1.63$ in the IHS in the MHD/kinetic code affects the outward-propagating ENA distribution, especially near the heliotail, therefore a more realistic description of the IHS plasma may be required to properly understand the contribution of the neutralized IHS plasma to the ribbon source region.

Interestingly, during slow SW, the high energy ribbon flux is slightly greater near the tail compared to the nose, opposite to the case at low ENA energies. At this ENA energy, the majority of ribbon ENAs originate from PUIs generated from outwardpropagating primary ENAs from the IHS (see bottom right panels of Fig. 3). As the SW speed increases, the component of fast ENAs from the supersonic SW increases near the nose of the heliosphere, dominating the ribbon flux.

We also note a few interesting characteristics of the ribbon flux originating from IHS primary ENAs. First, this flux is nearly independent of the SW speed, although it is consistently higher for fast SW. Second, while the ribbon flux originating from supersonic SW primary ENAs peaks near the nose of the heliosphere (largely due to the fact that most interstellar neutral atoms will charge exchange in the nose-ward propagating part of the supersonic SW), the component originating from IHS primary ENAs is nearly uniform around the ribbon, with a slight increase towards the heliotail. The competition of these components appears to control the relative ribbon intensity during different SW conditions. However, we point out that, in realistic SW conditions, the heliotail is comprised of a history of multiple solar cycles (see Zirnstein et al. 2015b, Fig. 2), and thus the ribbon spectrum may be influenced by primary ENAs originating from IHS plasma parcels of varying thermodynamic properties beyond our simulations. This should be taken into account when analyzing the ribbon flux from near the heliotail.

Figure 3 can be directly compared to Fig. 9 of Funsten et al. (2015), who analyzed the symmetry of the IBEX ribbon; however, note that Funsten et al. plot the IBEX ribbon flux and location as a function of angle from the ribbon symmetry axis, which lies within $\sim 40^{\circ}$ of the $B-V$ plane $\left(0^{\circ}\right.$ azimuth in our Fig. 3$)$. The IBEX ribbon flux appears to maximize close to the symmetry axis for 0.71 and $1.11 \mathrm{keV}$, relatively close to the $B-V$ plane (within $\sim 30^{\circ}$ ), similar to our results at low ENA energies for the case of slow SW, and high ENA energies during fast SW both in the absence of an asymmetric SW. At higher energies, the IBEX ribbon flux appears bimodal, likely due to the asymmetric SW, which is excluded in our study. Nevertheless, in all cases, our results appear to be symmetric about the $B-V$ plane. This suggests that, besides the possible influence of the latitudinal asymmetry of the SW, the hydrodynamic interaction of the SW with the LISM, centered on the trajectory of the heliosphere through the interstellar gas, is an important ordering process for the ribbon flux, as also found by Funsten et al. (2015).

\subsubsection{Comparing simulated and IBEX ribbon geometry}

The aim of this analysis is to determine if there is a significant dependence of the ribbon's geometric properties on the SW speed and ENA energy. Since the draping of the LISM magnetic field is a function of distance from the heliopause (e.g., Grygorczuk et al. 2011; Pogorelov et al. 2011; Zirnstein et al. 2015a), the energy of the parent SW and ENA distribution may affect the distances at which the majority of ribbon ENAs (observed at $1 \mathrm{au}$ ) originate. We are not attempting to reproduce the IBEX results, but rather attempt to understand the effects of SW speed and ENA energy on the geometry of the secondary ENA ribbon, in the absence of time-dependent and asymmetric SW effects.

To illustrate the effect of the draped LISM magnetic field on the ribbon, in Fig. 4 we show the angle between the lineof-sight view from IBEX and the LISM magnetic field vector, determined from our "intermediate" SW simulation, calculated at distances of 20 and 200 au radially outside the heliopause. We compute the locus of points defined by $\boldsymbol{B} \cdot \boldsymbol{r}=0$ (i.e., with angle $\sim 90^{\circ}$ ), and fit a circle to these points using the method described in Appendix A. The frame of reference of these plots is the same as those shown in Fig. 2. Close to the heliopause, the locus of points defined by $\boldsymbol{B} \cdot \boldsymbol{r}=0$ is highly non-circular, especially near the heliotail. In the example shown at 20 au outside the heliopause, ribbon ENAs generated at this distance will have an effective radius near $76.6^{\circ} \pm 4.2^{\circ}$ when viewed at 1 au (the uncertainty is large due to the deviation of the fit near the heliotail). The center of the ribbon is also offset from the pristine LISM field direction (plot center) by $\sim 7^{\circ}$. Farther from the heliopause, the locus defined by $\boldsymbol{B} \cdot \boldsymbol{r}=0$ is wider and more circular. For example, at 200 au outside the heliopause, the effective ribbon radius would be $83.5^{\circ} \pm 0.4^{\circ}$, and the center of the ribbon is closer to the pristine field direction (offset by $\sim 4^{\circ}$ ). Thus the geometric properties of the secondary ENA ribbon, such as its center, radius, and eccentricity, are determined by the superposition of ENAs produced at different distances from the heliopause. The energy of the parent SW and ENA distribution may affect the depth of the ribbon's source since higher energy ENAs can travel farther through the heliosheath before experiencing charge 
E. J. Zirnstein et al.: Effects of solar wind speed on energetic neutrals from the IBEX ribbon
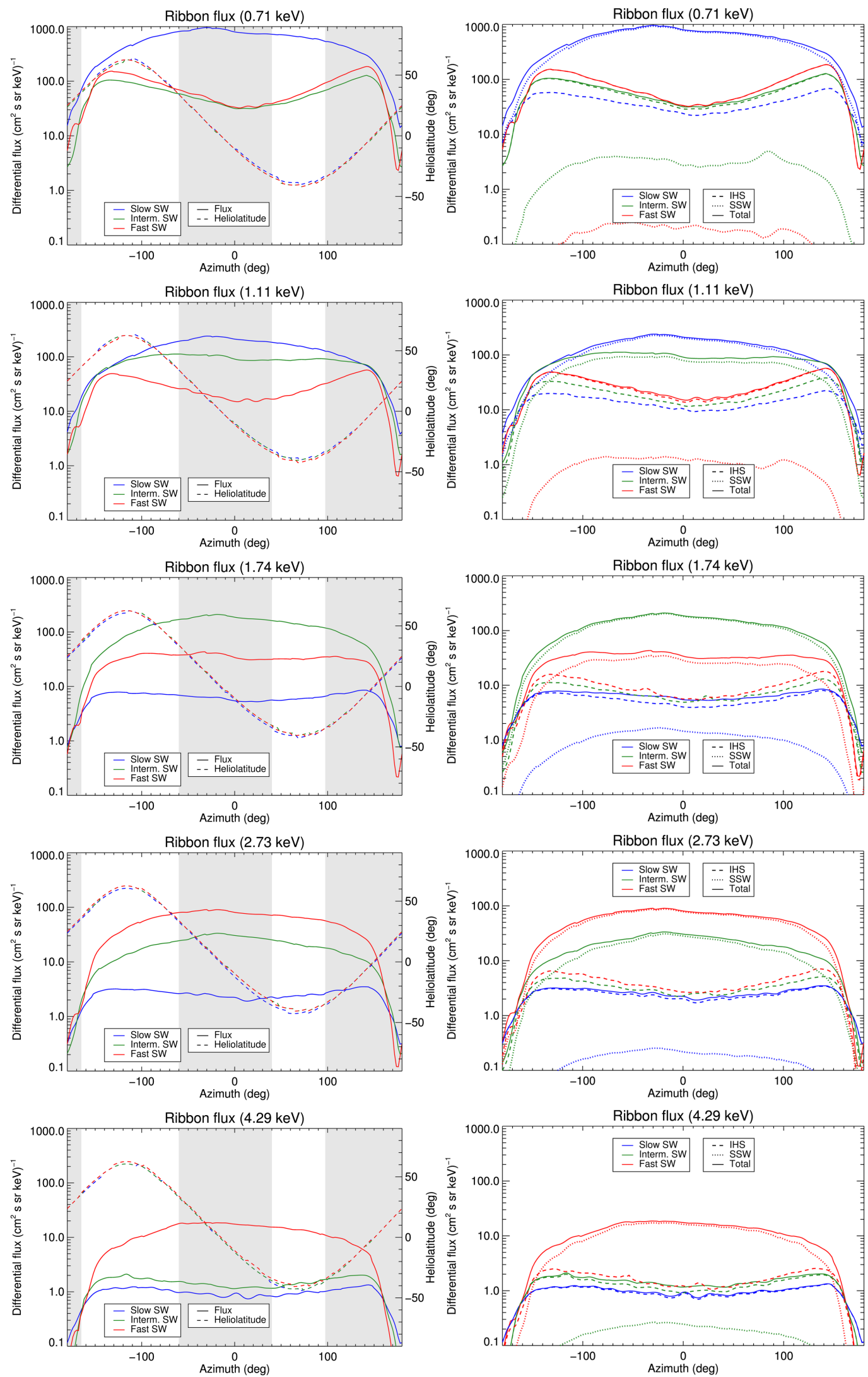

Fig. 3. Simulated ribbon flux from locations along the elliptical fits, as a function of azimuthal angle about the circularity plot center (negative to positive angle is counterclockwise in Fig. 2). In the left panels we show the ribbon flux at each energy (different rows) for the slow, intermediate, and fast SW (solid curves), as well as the heliolatitude location of the ribbon (dashed curves). The shaded regions correspond to locations of the ribbon lying within $\pm 35^{\circ}$ heliolatitude. Notice that the left $y$-axis is in $\log _{10}$ scaling. In the right panels we show the components of the ribbon flux that originated from outward-propagating, primary ENAs born in the IHS, the supersonic SW (SSW), and the total. 

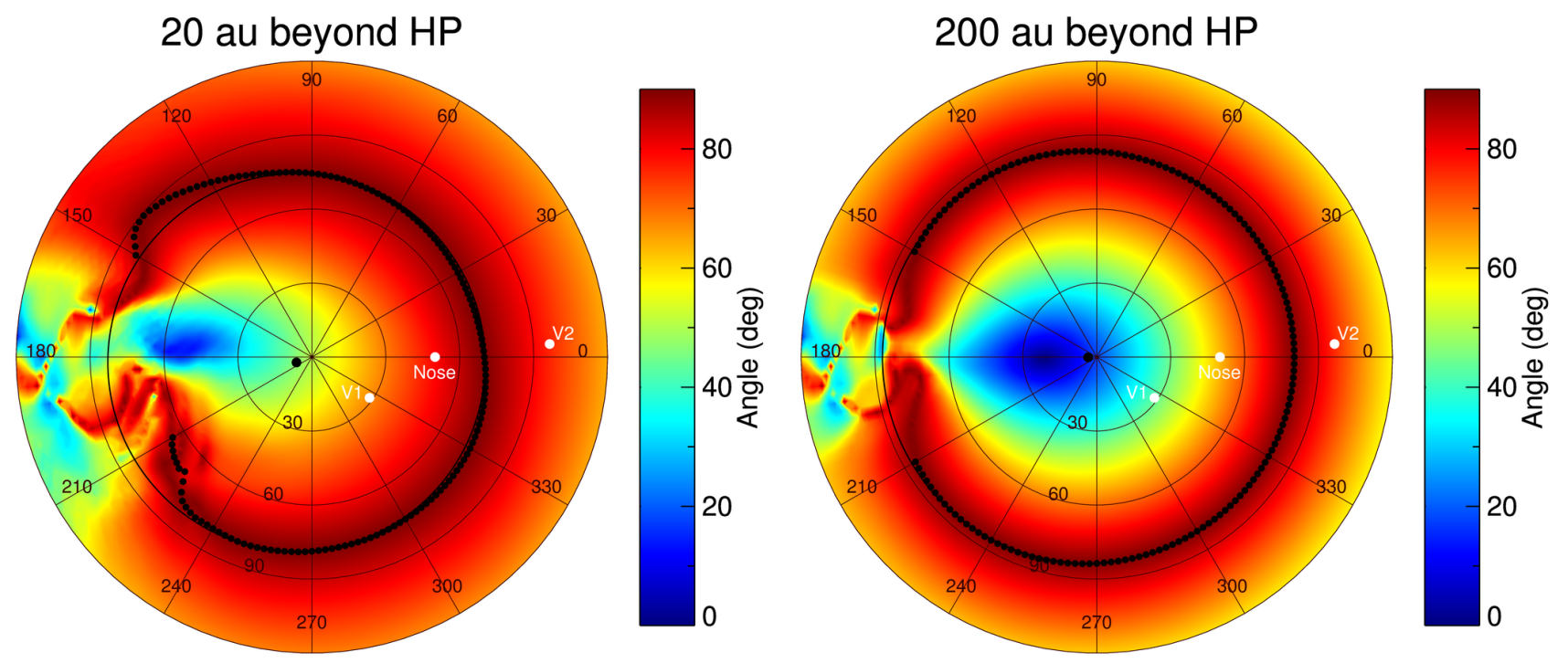

Fig. 4. Simulated angles between the line-of-sight view from IBEX and the local interstellar magnetic field vector, at distances of 20 au (left) and $200 \mathrm{au}$ (right) from the heliopause (HP). We show the locus of points defined by $\boldsymbol{B} \cdot \boldsymbol{r}=0$ (black dots), fit a circle to that locus of points (black curve), and plot the center of the fitted circle (black dot near the center of the plot). The frame of reference of these plots is the same as those shown in Fig. 2.

Table 2. Weighted averages of geometric properties.

\begin{tabular}{lcccc}
\hline \hline Parameters & Slow SW & Intermediate SW & Fast SW & IBEX \\
\hline Center longitude $\left(^{\circ}\right)$ & $213.5 \pm 0.2$ & $213.0 \pm 0.3$ & $213.1 \pm 0.3$ & $219.2 \pm 1.3$ \\
Center latitude $\left(^{\circ}\right)$ & $43.5 \pm 0.2$ & $43.6 \pm 0.3$ & $43.5 \pm 0.3$ & $39.9 \pm 2.3$ \\
Radius $\left({ }^{\circ}\right)$ & $80.6 \pm 0.5$ & $80.3 \pm 0.6$ & $79.8 \pm 0.6$ & $74.5 \pm 2.0$ \\
Eccentricity & $0.22 \pm 0.02$ & $0.24 \pm 0.02$ & $0.25 \pm 0.03$ & $0.31 \pm 0.08$ \\
Rotation angle $\left(^{\circ}\right)$ & $130 \pm 3$ & $121 \pm 1$ & $117 \pm 3$ & $95 \pm 15$ \\
\hline
\end{tabular}

Notes. The IBEX data are from Funsten et al. (2013). All results in this table are weighted over energy and circle/ellipse fits.

exchange (Möbius et al. 2013; Zirnstein et al. 2015a), which in turn may be revealed in the observed ribbon's geometry. We note that Isenberg et al. (2015) produced qualitatively similar results to our Fig. 4 from their passive model of the draped LISM magnetic field, where their locus defined by $\boldsymbol{B} \cdot \boldsymbol{r}=0$ is also larger at distances farther away from the heliosphere (for more details of their model, see Isenberg et al. 2015). It is also worth mentioning the analytic solution of the LISM magnetic field draped around the heliosphere provided by Röken et al. (2015), and further extended by Kleimann et al. (2016), may also be a useful tool for analyzing the draped LISM magnetic field in the context of the IBEX ribbon.

Overall, there only appears to be a small dependence of ribbon geometry on different SW speeds, and it is difficult to interpret in some cases because (1) the variations are small compared to the IBEX spatial resolution; or (2) the uncertainties are similar to the size of the variation. The final results of our analyses, weighted over IBEX-Hi energies, are shown in Table 2.

In Fig. 5, we show the derived ribbon centers as a function of ENA energy and SW speed, for circular and elliptical fits, for the simulation results and IBEX data. There is a small, visible trend in the slow and intermediate SW cases, where the higher energy ENA ribbon centers are generally closer to the pristine interstellar field direction (the IBEX ribbon center is assumed to be the pristine field direction in this paper), consistent with the idea that higher energy ENAs are more likely to originate from farther outside the heliopause (see Fig. 4). The low energy passbands detect a significant number of low energy ENAs coming from close outside the heliopause, while the high energy passbands detect a small number of ENAs originating from the high energy part (in the solar-inertial frame) of the neutralized slow SW distribution, coming farther away from the heliopause. This can ultimately produce a ribbon center closer to the pristine interstellar field direction. However, we note the variations are less than an IBEX pixel resolution of $6.5^{\circ} \mathrm{FWHM}$. During faster SW, this trend is less visible, and the centers nearly overlap within the uncertainties. Therefore, our results show that the center of the ribbon does not appear to change significantly with SW speed and ENA energy. The level at which the center of the ribbon is affected by the time dependence or asymmetry of the SW is left for a future study.

The ribbon centers, weighted over the IBEX-Hi energy passbands for uniform slow, intermediate, and fast SW (bottom right of Fig. 5) nearly overlap each other. While there is a clear offset $\left(\sim 5^{\circ}\right)$ between the simulated ribbon centers and the IBEX center/LISM field direction (due to the choice of $\boldsymbol{B}$, see Sect. 4), and they all lie close to the $B-V$ plane, there is no clear trend (e.g., linear) between the SW speed and the simulated ribbon center. Instead, this suggests that the draping of the interstellar magnetic field plays a dominant role in determining the angle between the ribbon center and pristine interstellar field direction, which is, in effect, determined by the curvature of the $\boldsymbol{B} \cdot \boldsymbol{r}=0$ surface in the vicinity of the heliosphere (Fig. 4), and that the line integration of ENAs masks the differences in the depths of their source. Also, in contrast to the IBEX observations in which the ribbon center was a slight function of ENA energy and the 

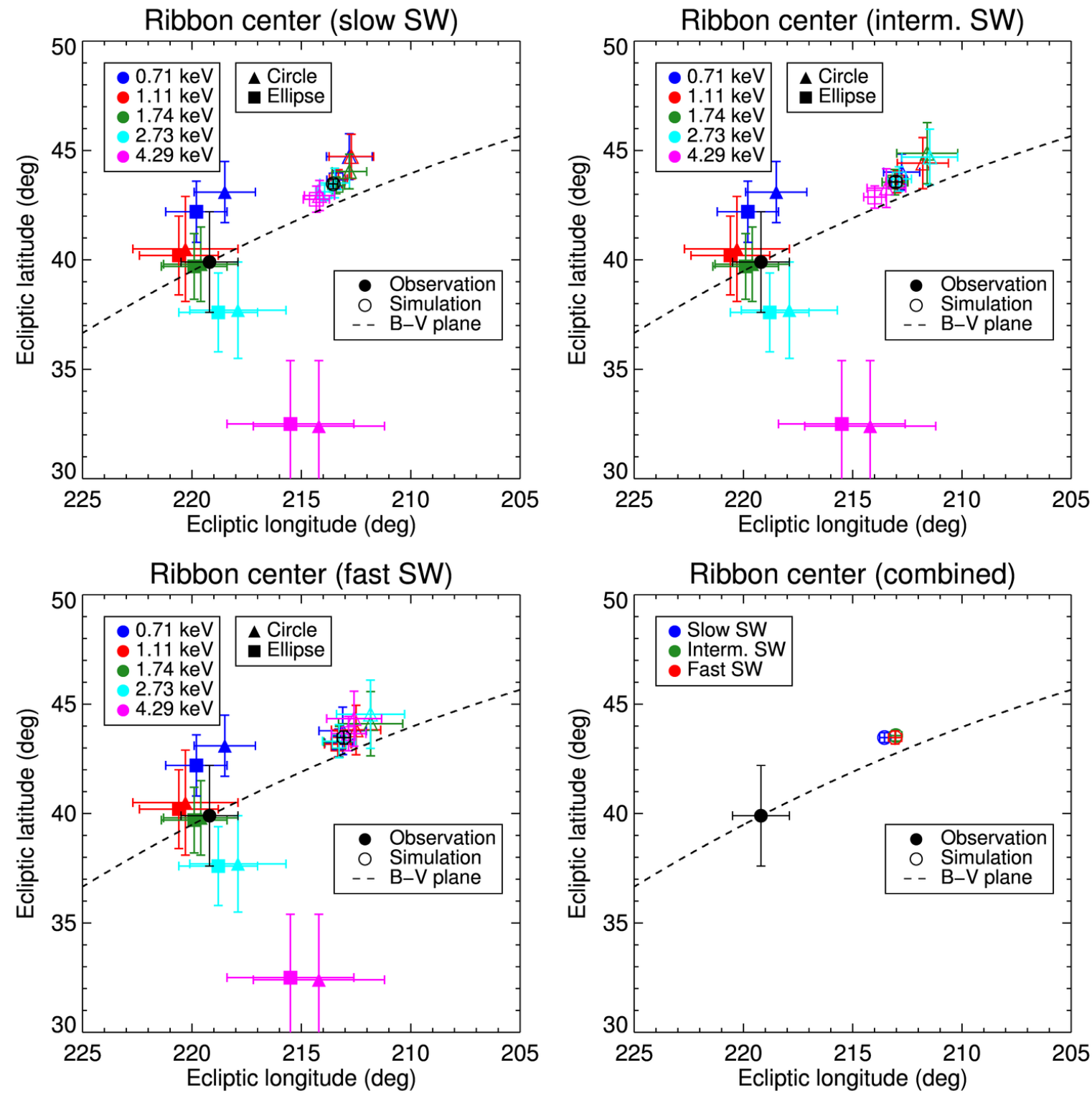

Fig. 5. Simulated ribbon centers for the slow (top left), intermediate (top right), and fast (bottom left) SW, and combined over all energies (bottom right). The simulation results are shown in open symbols, and the IBEX ribbon centers from Funsten et al. (2013) are shown in filled symbols, with the weighted centers (over energy and fit) shown in black symbols in the top row and bottom-left panel. In the bottom-right panel, the results are weighted over both circular and elliptical fits as well as energy. The interstellar field direction in the simulation overlaps the IBEX ribbon weighted center (black circle). We also show the $B-V$ plane as a black dashed curve, assuming the pristine interstellar magnetic field is nearly aligned with the IBEX ribbon center $\left(219.2^{\circ}, 39.9^{\circ}\right.$; Funsten et al. 2013).

location shifted generally perpendicular to the $B-V$ plane, the slow and intermediate SW simulation results indicate a small, systematic energy dependence of ribbon center location and in a direction along the $B-V$ plane (also see Isenberg et al. 2015). Differences in the ribbon centers derived from IBEX data and from simulation results may arise from, for example, influences of non-ribbon ENA emissions, such as spatial variation of the globally distributed flux, that are included in the analysis of the IBEX data. As stated earlier, non-uniform SW conditions, which are excluded in this study, may significantly impact the ribbon center location of the simulation results. This will be analyzed in more detail in a future study.

The simulated ribbon radius (Fig. 6, top panel) is $\sim 7 \%$ larger than the IBEX ribbon radius at every energy (although similar at $4.29 \mathrm{keV}$ ) for every simulated SW case. Considering that the radius shows little dependence on SW speed, this effect is likely due to our choice for the interstellar magnetic field vector. In fact, previous studies have shown that the radius of the IBEX ribbon is a function of the interstellar magnetic field strength and orientation (e.g., Grygorczuk et al. 2011; Heerikhuisen \& Pogorelov 2011; Ratkiewicz et al. 2012; Heerikhuisen et al. 2014), suggesting our choice of $3 \mu \mathrm{G}$ for the interstellar magnetic field strength is too large and the vector direction may be slightly offset. While this will be more carefully analyzed in a future study, this suggests $\sim 3 \mu \mathrm{G}$ as the upper limit for the interstellar magnetic field strength, consistent with some previous studies (e.g., Heerikhuisen \& Pogorelov 2011; Strumik et al. 2011; Ben-Jaffel \& Ratkiewicz 2012; Ben-Jaffel et al. 2013; Heerikhuisen et al. 2014). The simulated ribbon radii show no or only a small trend with SW speed. Considering their uncertainties, only the slow SW case appears to show a systematic increase as a function of ENA energy, however it is 

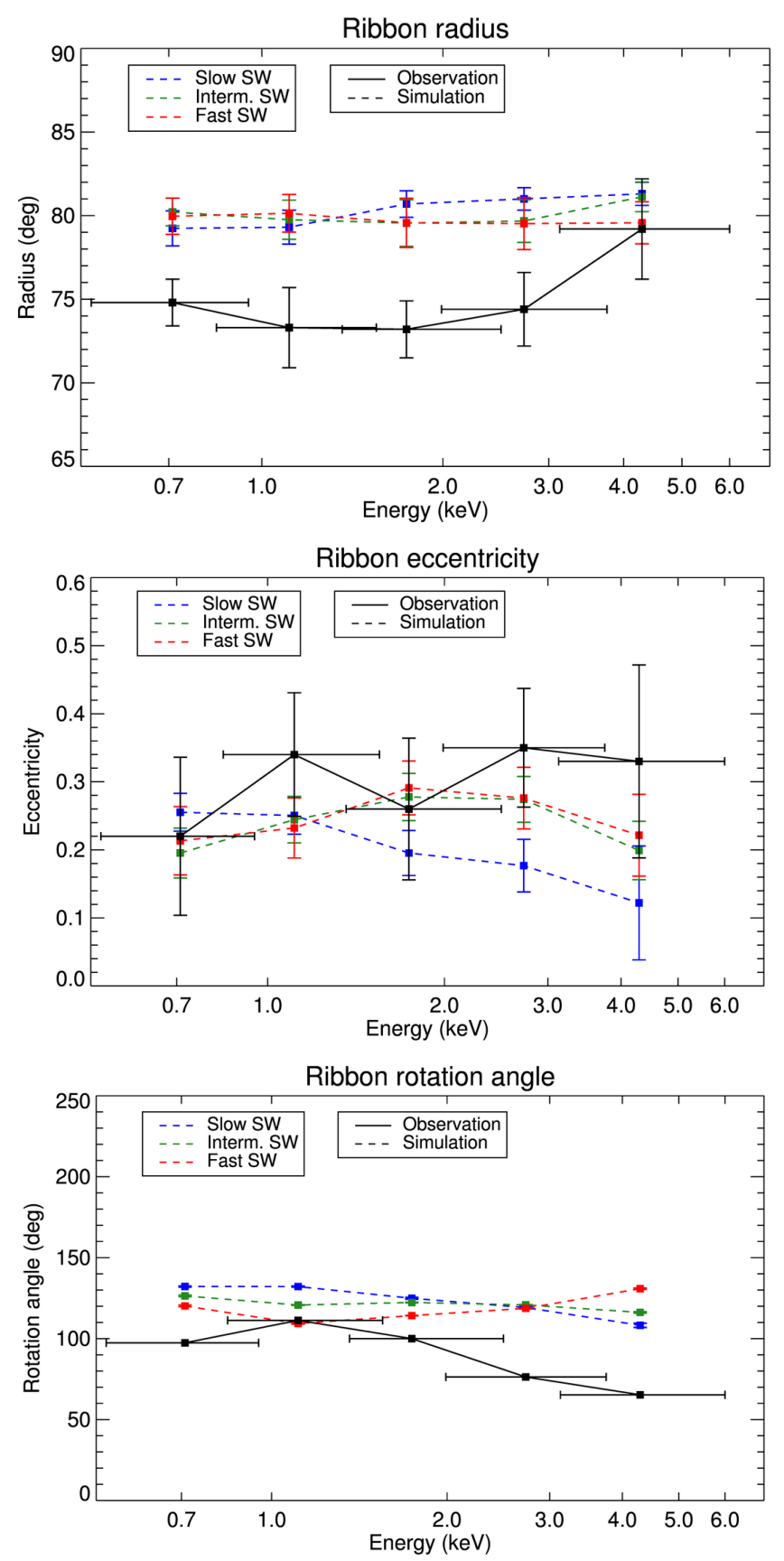

Fig. 6. Ribbon radius (top panel), eccentricity (middle panel) and semimajor axis rotation angle (bottom panel) for the simulation results (blue, green, and red dashed curves) and IBEX data (black solid curve). The rotation angle is defined as the counterclockwise angle between the semi-major axis of the fitted ellipse and $0^{\circ}$ in azimuth (see Fig. 3). We also show the uncertainties of the results and data (except for IBEX rotation angle uncertainties), including the FWHM energy ranges of the IBEX-Hi energy passbands (McComas et al. 2012b), which are also used in the simulation.

small compared to the IBEX angular resolution. In the case of faster SW, the radius appears to be independent of ENA energy, similar to the behavior of the ribbon center.

We may expect the IBEX ribbon radius to increase as a function of ENA energy, as is seen at $4.29 \mathrm{keV}$ in the IBEX data, due to higher energy ENAs originating farther away from the heliopause, producing a ribbon radius closer to a great circle. However, it is difficult to identify this trend in the simulation results since the radii do not vary more than $\sim 3^{\circ}$, which is approximately half of the IBEX angular resolution. Moreover, Zirnstein et al. (2015a) showed that while the ribbon source from secondary ENAs extends further into the LISM for higher ENA energies, the majority of the ribbon flux visible at 1 au comes from close to the heliopause (also see Heerikhuisen \& Pogorelov 2011). It is reasonable to suggest that the SW speed or ENA energy does not appear to significantly affect the simulated ribbon radius, except perhaps statistically at $4.29 \mathrm{keV}$, and we conclude that changes in uniform SW conditions will not significantly affect the IBEX ribbon radius. It is also possible that the increase in IBEX ribbon radius at $4.29 \mathrm{keV}$ is partially affected by the inclusion of source populations other than the ribbon, which are not included in our results.

The ribbon eccentricity is shown in the middle panel of Fig. 6. Analyzing trends in IBEX ribbon eccentricity as a function of energy is difficult, since changes in the data clearly lie within their uncertainties over all energies. While we can see some trends in the simulated ribbon eccentricity, such as a decrease in eccentricity above $\sim 1 \mathrm{keV}$ during slow SW, the uncertainties are too large to determine any statistically significant trend. Also, the ribbon eccentricity during intermediate and fast SW appears nearly identical. However, the weighted eccentricity of the IBEX ribbon is $0.31 \pm 0.08$ (Funsten et al. 2013), and the weighted eccentricities of the simulation results are $0.22 \pm$ $0.02,0.24 \pm 0.02$, and $0.25 \pm 0.03$, for the slow, intermediate, and fast SW cases, respectively. Therefore the simulated ribbon eccentricity in each case is lower than the IBEX ribbon (on the edge of their uncertainties), and characteristic of a more circular ribbon. This suggests that the higher eccentricity derived from IBEX data may be influenced by the latitudinal asymmetry of the SW, at least at the higher energies. However, since we exclude the latitudinal asymmetry of the SW in our analysis, this does not explain the fact that the simulation results do have some eccentricity, suggesting the draped interstellar magnetic field is responsible for a significant part of the IBEX ribbon eccentricity.

Similar to the previous properties, the simulated ribbon rotation angles of the semi-major axes of the elliptical fits (Fig. 6, bottom panel) show only a small dependence on SW speed and ENA energy. During slow SW, the rotation angle appears to maximize near $1 \mathrm{keV}$, and slightly decrease at higher ENA energies. During fast SW, the opposite occurs, where the rotation angle minimizes near $1 \mathrm{keV}$ and increases at higher ENA energies. During intermediate SW, the rotation angle appears nearly independent of the SW speed. It is interesting to note that the simulated ribbon rotation angle is between $\sim 100-140^{\circ}$ for every SW speed and ENA energy. Upon comparing their weighted averages $\left(130^{\circ} \pm 3^{\circ}, 121^{\circ} \pm 1^{\circ}, 117^{\circ} \pm 3^{\circ}\right.$, for slow, intermediate, and fast SW cases, respectively), we see an overall increase in the rotation angle, away from perpendicular to the $B-V$ plane, and away from the IBEX data $\left(95^{\circ} \pm 15^{\circ}\right)$. In particular, the ribbon is offset the most in the slow SW case $\left(34^{\circ} \pm 18^{\circ}\right)$, and less offset in faster SW. This discrepancy between the simulation and observation is likely due to our use of uniform SW boundary conditions. Also, Funsten et al. (2013) found that there is a significant dependence of the IBEX ribbon rotation angle on ENA energy. If the semi-major axis is related (but perpendicular) to the energy-dependent symmetry axis of the IBEX ribbon found by Funsten et al. (2015), and the unimodal/bimodal symmetry of the IBEX ribbon as a function of ENA energy is affected by the asymmetric SW, then it is reasonable to suggest that the rotation angle of the IBEX ribbon is also affected by the latitudinal asymmetry of the SW. Non-ribbon ENA emission features may lie within the region scribed by the ribbon and may 
also contribute to both non-circularity and asymmetry at different energies (Funsten et al. 2015).

We note that discrepancies between our results and the IBEX data may also be related to the fact that we included all azimuthal sectors around the ribbon (except near the heliotail) in our analysis of the ribbon's geometrical properties (see Fig. 2), but Funsten et al. (2013), although using similar requirements in choosing acceptable azimuthal sectors for their analysis of the IBEX data, excluded a larger number of sectors, mostly due to regions of low ribbon flux (relative to the background) or interference from magnetospheric observations, which are not issues in our simulations. Fitting circles and ellipses to different regions of the ribbon may have introduced some uncertainties in our comparisons.

\section{Conclusion}

In this paper we used simulations of the 3D heliosphere to estimate the dependence of the IBEX ribbon structure, position, and shape on the SW speed and ENA energy. We simulated the heliosphere for three separate SW conditions (uniform slow, intermediate, and fast SW), independent of time and latitude, with similar dynamic pressures (between $8 \%$ and $16 \%$ variation) and the same LISM boundary conditions, in order to understand the structural dependence of the ribbon on the SW speed and ENA energy. Since most of the secondary ENAs originate from primary ENAs produced in the supersonic SW ahead of the termination shock (except near the heliotail, see Fig. 3), the speed of the SW between $\sim 1$ au and the termination shock directly determines the primary (and secondary) ENA energy (assuming negligible diffusion in velocity space during the PUI phase in the OHS).

The intensity of the ribbon flux, as expected, was strongly dependent on the SW boundary conditions, with high intensity at low ENA energies during slow SW, and high intensity at high ENA energies during fast SW. It is clear that the latitudinaldependent structure of the ribbon, as a function of energy, is not seen in these results, due to the uniform SW boundary conditions. Considering the temporal evolution associated with the secondary emission process from SW input to detection at IBEX, the majority of ENAs detected during the first few years of IBEX observations at high latitudes likely originate from fast SW. Nevertheless, in the coming years, we expect that the low energy ribbon flux should increase and the high energy ribbon flux decrease at high latitudes. Even with the continued emission of fast SW at lower latitudes near solar maximum, the current solar cycle appears to be significantly less active than previous cycles, and we expect a significant drop to occur in the high energy ribbon flux, especially at $4.29 \mathrm{keV}$, compared to previous IBEX observations.

An interesting result of the simulation is that the symmetry of the simulated ribbon flux with respect to the $B-V$ plane depends on the SW speed and ENA energy. We have seen that the simulated ribbon flux is symmetric about the nose-heliotail vector, with higher intensity near the nose during slow SW conditions at low ENA energies, higher intensity near the heliotail at high ENA energies, and the roles reversed during fast SW conditions. The IHS plasma can contribute a significant number of primary ENAs to the OHS PUI source population, which may be visible during times when the average kinetic energy of the supersonic SW that produced those primary ENAs is sufficiently different from the energy of the observed ENAs. These results also indicate the importance of the ordering effect of the SW-LISM hydrodynamic interaction on the ribbon, and may help predict the evolution of the secondary ENA ribbon intensity with solar activity (also see Zirnstein et al. 2015b). However, more accurate comparisons of the absolute intensity of the ribbon should take into account the time-dependent SW.

The results of our geometry analyses (Sect. 3.2.2) suggest only a modest dependence of the ribbon center, radius, eccentricity, and rotation angle on SW speed and ENA energy. For instance, there appears to be a small dependence of the ribbon center on ENA energy during slow SW conditions, although with variations smaller than the size of an IBEX pixel. When we include faster SW speeds, differences in the ribbon center as a function of ENA energy are more difficult to discern, and the combined centers (weighted over energy) are nearly the same during different SW conditions. Our results show there is little detectable dependence of ribbon center on SW speed, suggesting that possible deviations in the ribbon center due to differences in the depth of ribbon source are largely independent of SW speed and ENA energy.

Similar to the ribbon center, the angular radius of the simulated ribbon shows only a slight dependence with ENA energy during slow SW. Also, compared to IBEX data (Funsten et al. 2013), the simulation results systematically overestimate the ribbon radius by $\sim 5^{\circ}$ at almost every energy and SW speed. This is likely due to our choice of the interstellar magnetic field strength and orientation, which have been shown to affect the IBEX ribbon radius (e.g., Grygorczuk et al. 2011; Heerikhuisen \& Pogorelov 2011; Ratkiewicz et al. 2012; Heerikhuisen et al. 2014). The simulated ribbon center is also offset by $\sim 5^{\circ}$ from the IBEX ribbon center, likely for the same reason. An analysis of the simulated ribbon flux circularity as a function of LISM magnetic field strength and orientation will be pursued in a future study.

Any dependence of the simulated ribbon eccentricity on SW speed is difficult to analyze due to the large uncertainties in the results. However, we can see that the results show an average eccentricity that is lower than the IBEX ribbon eccentricity, suggesting that the simulated ribbon is more circular. This is possibly due to the assumption of uniform SW conditions, which remove the bimodal structure of the high energy ribbon flux, possibly creating a more circular ribbon; also, our choice for the LISM magnetic field strength may be too large, resulting in a less-distorted interstellar magnetic field near the heliopause. The simulated ribbon rotation angle shows some systematic dependence on SW speed and ENA energy. It is statistically larger than the observed ribbon rotation angle during all SW cases, with a semi-major axis that is on average closer to the $B-V$ plane (assuming the IBEX ribbon center is near the pristine interstellar field direction).

According to our results, the draping of the LISM field around the heliopause appears to be an important driver for the observed IBEX ribbon structural and geometrical properties based on the secondary ENA mechanism. Since we excluded the effects of the time-dependent and latitudinal-dependent SW, the geometry of the ribbon does not appear to significantly depend on the SW speed. Moreover, the deviation of the simulated ribbon center, radius, eccentricity, and rotation angle from the data, compared to their uncertainties, indicate that these properties are key components in comparing the data and simulation results, and in determining the underlying processes that govern the ribbon.

Acknowledgements. This work was carried out as part of the IBEX mission, which is part of NASAs Explorer Program. Work at Los Alamos was performed under the auspices of the United States Department of Energy. J.H. 
acknowledges support from NASA grants NNX14AP24G, NNX12AH44G, NNX14AF43G, and NNX14AJ53G. E.Z. thanks George Livadiotis for helpful discussions.

\section{References}

Ben-Jaffel, L., \& Ratkiewicz, R. 2012, A\&A, 546, A78

Ben-Jaffel, L., Strumik, M., Ratkiewicz, R., \& Grygorczuk, J. 2013, ApJ, 779, 130

Burlaga, L. F., \& Ness, N. F. 2014, ApJ, 795, L19

Burlaga, L. F., Ness, N. F., Florinski, V., \& Heerikhuisen, J. 2014, ApJ, 792, 134

Burlaga, L. F., Florinski, V., \& Ness, N. F. 2015, ApJ, 804, L31

Bzowski, M., Kubiak, M. A., Möbius, E., et al. 2012, ApJS, 198, 12

Chalov, S. V., Alexashov, D. B., McComas, D., et al. 2010, ApJ, 716, L99

Christon, S. P., Williams, D. J., Mitchell, D. G., Frank, L. A., \& Huang, C. Y. 1989, J. Geophys. Res., 94, 13409

Desai, M. I., Allegrini, F. A., Dayeh, M. A., et al. 2012, ApJ, 749, L30

Desai, M. I., Allegrini, F. A., Bzowski, M., et al. 2014, ApJ, 780, 98

Ebert, R. W., McComas, D. J., Elliott, H. A., Forsyth, R. J., \& Gosling, J. T. 2009, J. Geophys. Res., 114, 1109

Fichtner, H., Scherer, K., Effenberger, F., et al. 2014, A\&A, 561, A74

Florinski, V., Zank, G. P., Heerikhuisen, J., Hu, Q., \& Khazanov, I. 2010, ApJ, 719, 1097

Frisch, P. C., Heerikhuisen, J., Pogorelov, N. V., et al. 2010, ApJ, 719, 1984

Funsten, H. O., Allegrini, F., Bochsler, P., et al. 2009a, Space Sci. Rev., 146, 75

Funsten, H. O., Allegrini, F., Crew, G. B., et al. 2009b, Science, 326, 964

Funsten, H. O., DeMajistre, R., Frisch, P. C., et al. 2013, ApJ, 776, 30

Funsten, H. O., Bzowski, M., Cai, D. M., et al. 2015, ApJ, 799, 68

Fuselier, S. A., Allegrini, F., Funsten, H. O., et al. 2009, Science, 326, 962

Gamayunov, K., Zhang, M., \& Rassoul, H. 2010, ApJ, 725, 2251

Giacalone, J., \& Jokipii, J. R. 2015, ApJ, 812, L9

Grygorczuk, J., Ratkiewicz, R., Strumik, M., \& Grzedzielski, S. 2011, ApJ, 727, L48

Grzedzielski, S., Bzowski, M., Czechowski, A., et al. 2010, ApJ, 715, L84

Heerikhuisen, J., \& Pogorelov, N. V. 2010, in ASP Numerical Modeling of Space Plasma Flows: ASTRONUM-2009, ed. N. V. Pogorelov, E. Audit, \& G. P. Zank (San Francisco, CA: ASP), Conf. Ser., 429, 227

Heerikhuisen, J., \& Pogorelov, N. V. 2011, ApJ, 738, 29

Heerikhuisen, J., Florinski, V., \& Zank, G. P. 2006a, J. Geophys. Res., 111, 6110

Heerikhuisen, J., Florinski, V., Zank, G. P., \& Pogorelov, N. V. 2006b, in Numerical Modeling of Space Plasma Flows: ASTRONUM-2006, ed. G. P. Zank \& N. V. Pogorelov (San Francisco, CA: ASP), ASP Conf. Ser., 359, 251

Heerikhuisen, J., Pogorelov, N. V., Florinski, V., Zank, G. P., \& Kharchenko, V. 2009, in Numerical Modeling of Space Plasma Flows: ASTRONUM-2008, eds. N. V. Pogorelov, E. Audit, P. Colella, \& G. P. Zank (San Francisco, CA: ASP), ASP Conf. Ser., 406, 189

Heerikhuisen, J., Pogorelov, N., \& Zank, G. 2010a, in Pickup Ions throughout the Heliosphere and Beyond: Proc. 9th Annual International Astrophysics Conference, eds. J. Le Roux, G. P. Zank, A. J. Coates, \& V. Florinski (Melville: NYAIP), AIP Conf. Ser., 1302, 98

Heerikhuisen, J., Pogorelov, N. V., Zank, G. P., et al. 2010b, ApJ, 708, L126

Heerikhuisen, J., Pogorelov, N., \& Zank, G. 2012, in Physics of the Heliosphere: A 10 Year Retrospective: Proc. 10th Annual Astrophysics Conference, eds. J. Heerikhuisen, G. Li, N. Pogorelov, \& G. Zank (Melville, NY: AIP), AIP Conf. Ser., 1436, 221

Heerikhuisen, J., Zirnstein, E. J., Funsten, H. O., Pogorelov, N. V., \& Zank, G. P. 2014, ApJ, 784, 73

Isenberg, P. A. 2014, ApJ, 787, 76

Isenberg, P. A., Forbes, T. G., \& Möbius, E. 2015, ApJ, 805, 153

Kleimann, J., Röken, C., Fichtner, H., \& Heerikhuisen, J. 2016, ApJ, 816, 29

Kucharek, H., Fuselier, S. A., Wurz, P., et al. 2013, ApJ, 776, 109

Lindsay, B. G., \& Stebbings, R. F. 2005, J. Geophys. Res., 110, 12213
Liu, K., Möbius, E., Gary, S. P., \& Winske, D. 2012, J. Geophys. Res., 117, 10102

Livadiotis, G., \& McComas, D. J. 2009, J. Geophys. Res., 114, 11105

Livadiotis, G., \& McComas, D. J. 2013, Space Sci. Rev., 175, 183

Markwardt, C. B. 2009, in Astronomical Data Analysis Software and Systems XVIII, eds. D. A. Bohlender, D. Durand, \& P. Dowler, ASP Conf. Ser., 411, 251

McComas, D. J., Ebert, R. W., Elliott, H. A., et al. 2008, Geophys. Res. Lett., 35,18103

McComas, D. J., Allegrini, F., Bochsler, P., et al. 2009, Science, 326, 959

McComas, D. J., Bzowski, M., Frisch, P., et al. 2010, J. Geophys. Res., 115, 9113

McComas, D. J., Alexashov, D., Bzowski, M., et al. 2012a, Science, 336, 1291

McComas, D. J., Dayeh, M. A., Allegrini, F., et al. 2012b, ApJS, 203, 1

McComas, D. J., Angold, N., Elliott, H. A., et al. 2013a, ApJ, 779, 2

McComas, D. J., Dayeh, M. A., Funsten, H. O., Livadiotis, G., \& Schwadron, N. A. 2013b, ApJ, 771, 77

McComas, D. J., Allegrini, F., Bzowski, M., et al. 2014a, ApJS, 213, 20

McComas, D. J., Lewis, W. S., \& Schwadron, N. A. 2014b, Rev. Geophys., 52, 118

McComas, D. J., Bzowski, M., Fuselier, S. A., et al. 2015, ApJS, 220, 22

Möbius, E., Bochsler, P., Bzowski, M., et al. 2012, ApJS, 198, 11

Möbius, E., Liu, K., Funsten, H., Gary, S. P., \& Winske, D. 2013, ApJ, 766, 129

Pogorelov, N. V., Zank, G. P., Borovikov, S. N., et al. 2008, in Numerical Modeling of Space Plasma Flows: ASTRONUM-2007, eds. N. V. Pogorelov, E. Audit, \& G. P. Zank (San Francisco, CA: ASP), ASP Conf. Ser., 385, 180 Pogorelov, N. V., Borovikov, S. N., Zank, G. P., \& Ogino, T. 2009, ApJ, 696, 1478

Pogorelov, N. V., Heerikhuisen, J., Zank, G. P., et al. 2011, ApJ, 742, 104

Ratkiewicz, R., Strumik, M., \& Grygorczuk, J. 2012, ApJ, 756, 3

Ripken, H. W., \& Fahr, H. J. 1983, A\&A, 122, 181

Röken, C., Kleimann, J., \& Fichtner, H. 2015, ApJ, 805, 173

Schwadron, N. A., \& McComas, D. J. 2013, ApJ, 764, 92

Schwadron, N. A., Bzowski, M., Crew, G. B., et al. 2009a, Science, 326, 966

Schwadron, N. A., Crew, G., Vanderspek, R., et al. 2009b, Space Sci. Rev., 146, 207

Schwadron, N. A., Moebius, E., Fuselier, S. A., et al. 2014, ApJS, 215, 13

Siewert, M., Fahr, H.-J., McComas, D. J., \& Schwadron, N. A. 2013, A\&A, 551, A58

Smith, E. J., Balogh, A., Forsyth, R. J., \& McComas, D. J. 2001, Geophys. Res. Lett., 28, 4159

Sokół, J. M., Bzowski, M., Tokumaru, M., Fujiki, K., \& McComas, D. J. 2013, Sol. Phys., 285, 167

Strumik, M., Ben-Jaffel, L., Ratkiewicz, R., \& Grygorczuk, J. 2011, ApJ, 741, L6

Summerlin, E. J., Viñas, A. F., Moore, T. E., Christian, E. R., \& Cooper, J. F. 2014, ApJ, 793, 93

Sylla, A., \& Fichtner, H. 2015, ApJ, 811, 150

Treumann, R. A. 1999, Phys. Scr., 59, 204

Vasyliunas, V. M. 1968, J. Geophys. Res., 73, 2839

Zank, G. P., Heerikhuisen, J., Pogorelov, N. V., Burrows, R., \& McComas, D. 2010, ApJ, 708, 1092

Zank, G. P., Hunana, P., Mostafavi, P., \& Goldstein, M. L. 2014, ApJ, 797, 87

Zhou, X., \& Smith, E. J. 2009, J. Geophys. Res. (Space Phys.), 114, 3106

Zirnstein, E., Heerikhuisen, J., \& Pogorelov, N. 2012, in AIP Space Weather: The Space Radiation Environment: Proc. 11th Annual International Astrophys. Conf., eds. Q. Hu, G. Li, G. P. Zank, et al. (Melville, NY: AIP), Conf. Ser., 1500, 262

Zirnstein, E. J., Heerikhuisen, J., McComas, D. J., \& Schwadron, N. A. 2013, ApJ, 778, 112

Zirnstein, E. J., Heerikhuisen, J., Zank, G. P., et al. 2014, ApJ, 783, 129

Zirnstein, E. J., Heerikhuisen, J., \& McComas, D. J. 2015a, ApJ, 804, L22

Zirnstein, E. J., Heerikhuisen, J., Pogorelov, N. V., McComas, D. J., \& Dayeh, M. A. 2015b, ApJ, 804, 5 


\section{Appendix A: Fitting a circle/ellipse to the simulated ribbon}

The simulated ribbon flux at each energy passband is fit to a circle and ellipse using a method similar to Funsten et al. (2013). First, we rotate the simulated ribbon flux into a ribboncentered coordinate system (centered on $\left(219.2^{\circ}, 39.9^{\circ}\right)$ ), and fit a Gaussian function,

$F_{\mathrm{G}}=A_{\mathrm{G}}+B_{\mathrm{G}} \times \exp \left(-\frac{\left(r-r_{\mathrm{c}}\right)^{2}}{2 \sigma_{\mathrm{G}}^{2}}\right)$,

to the flux as a function of angular distance $r$ from the center of the plot for each azimuthal sector (counterclockwise about the plot center, in $2^{\circ}$ increments), where $B_{\mathrm{G}}$ is the peak intensity of the Gaussian, $r_{\mathrm{c}}$ is the location of the Gaussian center and thus of the maximum flux, $\sigma_{\mathrm{G}}$ is the standard deviation, and $A_{\mathrm{G}}$ is the baseline flux. Similar to the analysis of Funsten et al. (2013), we fit the Gaussian function to the simulated ENA ribbon flux in a $42^{\circ}$ range, initially centered on the pixel of maximum flux. We also ignore certain azimuthal sections in our analyses. First, we ignore sections between $150^{\circ}$ and $210^{\circ}$ (see Fig. 2), in the immediate vicinity of the heliotail, which is a feature that is independent of the ribbon, in a similar way to what is observed in the IBEX data (McComas et al. 2013b). Second, similar to Funsten et al. (2013), we ignore azimuthal sections that produced uncertainties greater than $2^{\circ}$ in determining the location of maximum flux from the Gaussian fit $\left(r_{\mathrm{c}}\right)$ for $0.71-2.73 \mathrm{keV}$, and ignore sections with uncertainties greater than $3^{\circ}$ for $4.29 \mathrm{keV}$. Third, similar to Funsten et al. (2013), we ignore sections where $B_{\mathrm{G}} /\left|A_{\mathrm{G}}\right|<0.05$, i.e., regions of low flux (we specify $\left|A_{\mathrm{G}}\right|$ since $A_{\mathrm{G}}$ may be slightly negative when the background flux is very small - in our case we do not include flux from the IHS). In our simulations, this criterion largely applies to the vicinity of the heliotail, which is already excluded. The results of these fits give us the angular locations $r_{\mathrm{c}}$ of the maximum ribbon flux as a function of angular distance from the center $(r)$ for each $2^{\circ}$ azimuthal sector, and the 1-sigma uncertainties of these locations. The locations of accepted maximum flux locations are shown as black dots in Fig. 2. For all cases, the accepted locations of maximum flux span the complete circular ribbon except in the vicinity of the heliotail.

Next, we fit a circle to the location $r_{\mathrm{c}}$ of maximum flux at each azimuthal sector using Levenberg-Marquardt least-squares minimization (Markwardt 2009). The uncertainties in determining $r_{\mathrm{c}}$ from the Gaussian fit (not $\sigma_{\mathrm{G}}$ ) are used as weights for the circle fits, where the squared residuals in the minimization are weighted by $\sigma^{-2}$. One result of this circular fit is the center of the circle weighted by the uncertainties in the maximum flux locations; a second result is the mean circle radius, which is derived by computing the mean distance of each maximum flux location from the derived circle center. The uncertainty of the center and radius is found by computing the standard deviation of the maximum flux locations $r_{\mathrm{c}}$ from the mean radius.

We also fit the ribbon to an ellipse using a similar procedure. The center and semi-major/minor axis lengths are found by the minimization scheme (weighted by the uncertainties in the maximum flux locations $r_{\mathrm{c}}$ ), and their uncertainties are found by computing the standard deviation of the maximum flux locations from the derived elliptical fit center. The eccentricity is computed using the derived semi-major/minor axes, with an uncertainty computed by propagating the errors of the axis lengths. The rotation angle, defined as the counterclockwise angle between the semi-major axis and $0^{\circ}$ in azimuth (the $B-V$ line), and its uncertainty are also derived from the minimization scheme. The results of this analysis are shown in Sect. 3.2. 